Internist 2020 61:690-698

https://doi.org/10.1007/s00108-020-00814-z

Online publiziert: 27. Mai 2020

(C) Der/die Autor(en) 2020

\section{Redaktion}

M. Hallek, Köln

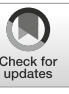

\author{
H.-G. Rammensee ${ }^{1,2,3} \cdot$ M. W. Löffler ${ }^{1,2,3,4,5} \cdot$ J. S. Walz ${ }^{1,2,3,7,8} \cdot$ C. Bokemeyer ${ }^{6} \cdot$ \\ S. P. Haen ${ }^{1,2,6} \cdot$ C. Gouttefangeas ${ }^{1,2,3}$ \\ 'Interfakultäres Institut für Zellbiologie (IFIZ), Abteilung Immunologie, Eberhard Karls Universität \\ Tübingen, Tübingen, Deutschland \\ ${ }^{2}$ Deutsches Konsortium für Translationale Krebsforschung (DKTK) am Deutschen \\ Krebsforschungszentrum (DKFZ), Partnerstandort Tübingen, Tübingen, Deutschland \\ ${ }^{3}$ Exzellenzcluster iFIT (EXC2180) „Individualisierung von Tumortherapien durch molekulare Bildgebung \\ und funktionelle Identifizierung therapeutischer Zielstrukturen", Tübingen, Deutschland \\ ${ }^{4}$ Abteilung für Allgemeine, Viszeral- und Transplantationschirurgie, Universitätsklinikum Tübingen, \\ Tübingen, Deutschland \\ ${ }^{5}$ Abteilung Klinische Pharmakologie, Universitätsklinikum Tübingen, Tübingen, Deutschland \\ ${ }^{6}$ Zentrum für Onkologie, II. Medizinische Klinik (Onkologie, Hämatologie, Knochenmarktransplantation \\ mit Abteilung für Pneumologie), Universitätsklinikum Hamburg-Eppendorf, Hamburg, Deutschland \\ ${ }^{7}$ Medizinische Klinik II für Hämatologie, Onkologie, Immunologie und Rheumatologie, \\ Universitätsklinikum Tübingen, Tübingen, Deutschland \\ ${ }^{8}$ Klinische Kooperationseinheit (KKE) für Translationale Immunologie, Universitätsklinikum Tübingen, \\ Tübingen, Deutschland
}

\title{
Tumorvakzinierung - therapeutische Vakzinierung gegen Krebs
}

(- Abb. 1), so dass diese von den T-Zellen erkannt werden können. Dies führt beispielsweise im Falle einer Virusinfektion der Zelle in der Regel zur Aktivierung virusspezifischer T-Zellen, die die infizierte Zelle dann abtöten.

Im Normalfall präsentiert jede Körperzelle zehntausend oder mehr Peptide aus Tausenden von zellulären Proteinen auf ihren HLA-Klasse-I-Molekülen. Diese Peptide entstehen in der Zelle durch enzymatischen Abbau der zellulären Proteine im Rahmen des normalen Stoffwechsels; der Großteil dieser Peptide wird bis zu einzelnen Aminosäuren abgebaut, eine kleine Auswahl dieser Peptide wird jedoch von den HLA-Molekülen im endoplasmatischen Retikulum gebunden und an die Zelloberfläche gebracht (• Abb. 1). Der Komplex aus HLAMolekül und Peptid kann dann von den T-Zellen erkannt werden. Im Normalfall, das heißt, wenn keine pathologischen Peptide darunter sind, passiert nichts, da die T-Zellen gegen die normalen körpereigenen Peptide tolerant sind. Im Falle einer Virusinfektion dagegen sind unter den Tausenden von HLA-präsentierten
Peptiden auch einige aus Virusproteinen, die dann von den T-Zellen als fremd erkannt werden können.

\section{\) Tumorspezifische Peptide wären ideale Kandidaten für eine therapeutische Vakzinierung}

Im Falle von Krebserkrankungen ist die Sachlage komplexer, da alle Proteine der Krebszelle grundsätzlich körpereigen sind. Was hier von T-Zellen - nicht als fremd, aber als pathologisch verändert - erkannt werden kann, sind beispielsweise sogenannte mutierte Neoantigene, das sind Peptide, die aus einer tumorspezifischen Mutation hervorgehen und damit eine andere Aminosäuresequenz aufweisen als das entsprechende normale Protein. Man geht davon aus, dass insbesondere solche Neoantigene durch die Immuncheckpointinhibitor(ICI)vermittelte T-Zell-Antwort erkannt werden, da unter anderem die Ansprechrate von Tumoren tendenziell mit der Zahl der Mutationen auf DNA-Ebene korreliert [6]. 


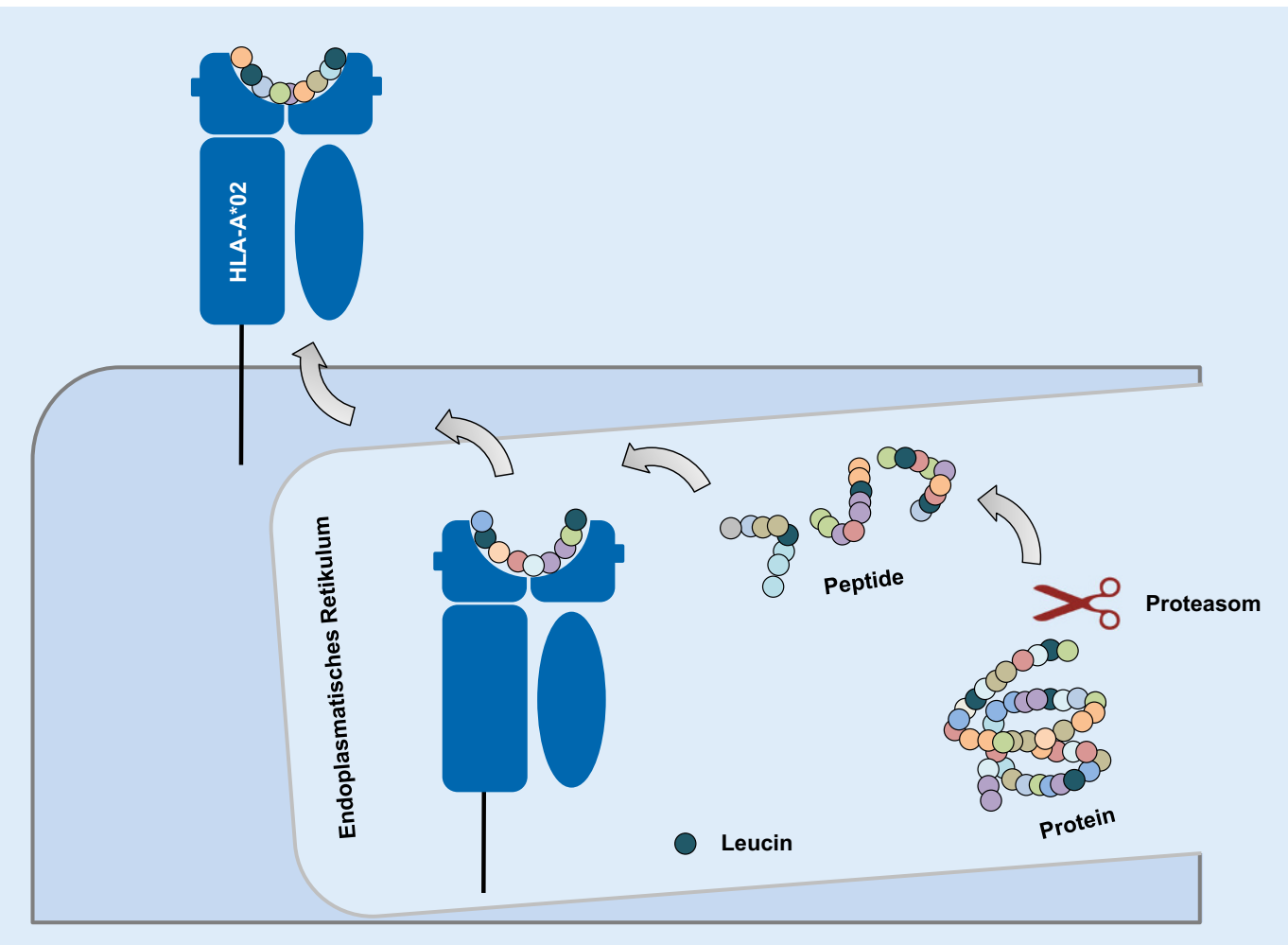

Abb. $1<$ Peptidbeladung und -präsentation auf HLAMolekülen. Grafische Darstellung der Prozessierung, HLA-Beladung und -Präsentation von passenden Peptiden („Motivpeptiden") auf der Zelloberfläche. HLA Humanes Leukozytenantigen

Darüber hinaus präsentieren Tumorzellen viele weitere tumorspezifische Peptide, die keine Mutationen aufweisen, aber auf normalen Zellen nicht vorkommen [27]. Eine solche krebsspezifische Peptidpräsentation kann viele unterschiedliche Ursachen haben [18], mehrheitlich sind diese aber noch nicht hinreichend erforscht.

Tumorspezifische Peptide - unabhängig davon, ob sie durch Mutation oder andere zelluläre Ereignisse entstehen wären ideale Kandidaten für eine therapeutische Vakzinierung. Dabei gibt es jedoch zwei maßgebliche Hindernisse, die bisher einen wesentlichen Erfolg verhindert haben:

- In jedem Menschen sind diese Peptide anders, und zwar sowohl aufgrund der Verschiedenheit unterschiedlicher maligner Zellen als auch wegen des ausgeprägten Polymorphismus der HLA-Gene.

- In klinischen Studien ist es bisher praktisch nicht gelungen, durch Vakzinierung bei Patienten effektive T-Zell-Antworten gegen solche Peptide hervorzurufen, vermutlich wegen des Fehlens geeigneter Adjuvanzien.

\section{Das System der humanen Leukozytenantigene und die Antigenpräsentation}

Das HLA-System ist bekannt wegen der Notwendigkeit der HLA-Übereinstimmung im Rahmen von Transplantationen, insbesondere bei hämatopoetischen Stammzellen. Die physiologische Funktion von HLA-Molekülen ist aber, Antigene für die Erkennung durch T-Zellen zu präsentieren (• Abb. 1). Fast alle Zellen eines Menschen exprimieren HLAMoleküle der Klasse I, das sind HLA-A, HLA-B und HLA-C. Diese HLA-Gene sind äußerst polymorph; für jeden der drei Loci sind Tausende von verschiedenen Allelen bekannt, insgesamt derzeit über 18.000 [25]. Die meisten Menschen sind für diese Loci heterozygot und exprimieren zwei verschiedene Allelprodukte, etwa HLA-A ${ }^{\star} 02$ (das häufigste Allel) und HLA-A*11. HLAKlasse-I-Moleküle präsentieren zelluläre Peptide, meist aus 9 Aminosäuren, mit bestimmten Sequenzeigenschaften („Motiv“); HLA-A*02-Peptide haben beispielsweise die Aminosäure Leucin oder Isoleucin häufig an Position 2 und 9. HLA-A ${ }^{\star} 11$-Peptide weisen hingegen oft aliphatische Aminosäuren an Position 2 auf, aber ein Lysin oder Arginin an Position 9. Solche HLA-Klasse-I-präsentierten Peptide werden von $\mathrm{CD}^{+}$-TZellen erkannt. Einen visuellen Eindruck von der funktionellen Vielfalt der HLAMoleküle bei verschiedenen Menschen vermittelt • Abb. 2.

\section{》) Jeder Mensch präsentiert eine individuelle Stichprobe des zellulären Peptidspektrums auf den HLA-Molekülen}

Diese Zusammenhänge sind für die $\mathrm{Su}$ che nach krebsspezifischen Antigenen von großer Bedeutung, da jeder Mensch eine individuelle Stichprobe des zellulären Peptidspektrums auf den HLAMolekülen zeigt und so für seine eigenen T-Zellen erkennbar macht. Damit ist beispielsweise $\mathrm{zu}$ erklären, warum bei vielen Tumoren das Gen KRAS als „onkogener Driver" mutiert ist, aber nur selten als Peptid auf HLA präsentiert wird und ebenso warum von Tausenden von Mutationen, etwa beim Melanom, nur ein kleiner Anteil auf den HLA-Mo- 
lekülen des Patienten präsentiert wird [2]. Das Gleiche gilt außerdem für tumorspezifische Peptide, die nicht von Mutationen abgeleitet sind.

Die HLA-Klasse-II-Moleküle (HLADR, -DQ und -DP) sind zwar mit etwas über 7000 Allelen nicht ganz so polymorph und in ihren Peptidmotiven etwas weniger strikt. Das führt aber ebenfalls dazu, dass über HLA-Klasse II nur eine Auswahl der tumorspezifischen Proteinveränderungen den T-Zellen präsentiert werden kann. HLA-Klasse-II-präsentierte Peptide werden von $\mathrm{CD}^{+}{ }^{-}$-T-Zellen erkannt.

In der Entwicklung von Strategien zur therapeutischen Tumorvakzinierung macht dieser Umstand ein personalisiertes Vorgehen erforderlich, um geeignete tumorspezifische HLA-Peptide zu charakterisieren.

\section{Identifikation tumor- spezifischer Peptide}

Gängiges Vorgehen ist es, aus Proteinen, die man für krebsspezifisch hält, über die auf den entsprechenden HLA-Motiven basierenden Vorhersagemethoden [4] in silico die für den jeweiligen Patienten passenden Peptide zu bestimmen. Diese müssten dann eigentlich mit handfesten Methoden - entweder mit T-Zell-Tests oder mittels Massenspektrometrie - bestätigt werden, um sicherzustellen, dass die Vorhersagen korrekt waren. Wenn dies unterbleibt, ist die Wahrscheinlichkeit hoch, dass im Patienten T-Zellen induziert werden, die zwar kräftig mit solchen Peptiden reagieren, aber völlig unnütz sind, da die Zielstrukturen auf den Tumorzellen überhaupt nicht vorhanden sind.

Die ersten tumorspezifischen Peptide wurden gefunden, indem in Patienten spontan entstandene T-Zellen auf ihre Spezifität hin untersucht wurden. Dabei wurden unter anderem die sogenannten Cancer-testis-Antigene entdeckt, die zwar in vielen Tumoren exprimiert werden, jedoch wegen des HLA-Polymorphismus in jedem Menschen mit unterschiedlichen Peptiden. Ebenso wurden Differenzierungsantigene entdeckt, die zwar nicht tumor-, sondern gewebespezifisch sind, aber wie beispielsweise bei prostataspezifischen Antigenen sonst nur auf „entbehrlichen“ Zellen vorkommen.

Die derzeit verfügbare rationale $\mathrm{Me}$ thode der Wahl ist jedoch, die Tumorzellen eines Patienten mittels Massenspektrometrie direkt auf HLA-präsentierte Peptide hin zu untersuchen, die auf normalem Gewebe nicht vorkommen. Dashört sich einfach an und ist es im Prinzip auch, allerdings ist es unmöglich, vom selben Patienten Proben aus (allen) Normalgeweben zu gewinnen. Ein Behelf ist es aber, möglichst die Gesamtheit aller HLA-präsentierten Peptide aller Gewebe von vielen Menschen mit allen oder zumindest mit den häufigsten HLA-Allelen zu bestimmen. Mit einer solchen - sehr großen - Datenbank ist es dann möglich, neu gefundene Peptide auf Tumorzellen einer gegebenen HLA-Typisierung als tumorspezifisch zuzuordnen. Dann muss noch getestet werden, ob im betreffenden Patienten T-Zellen mit passendem Rezeptor existieren. Das ist häufig tatsächlich der Fall [14].

\section{Induktion einer effektiven Immunantwort im Patienten}

Was die T-Zellen erkennen sollen, sind immer HLA-präsentierte Peptide, mit denen man zum einen direkt immunisieren kann. Es gibt aber zum anderen zahlreiche komplexere Antigenzubereitungen, in denen die tumorspezifischen Antigene enthalten sind (DNA, RNA, rekombinante Proteine, Viruskonstrukte, bakterielle Konstrukte), sowie undefinierte tumorzellbasierte Zubereitungen. In jedem Fall stellt sich das Problem des Applikationswegs und der Wahl eines geeigneten Adjuvans. Sehr effiziente Wege sind hier Viruskonstrukte, die aber das Problem aufweisen, auch Immunantworten gegen den Vektor zu induzieren, RNA-Formulierungen [1, 23], die i.v. oder i.m. appliziert werden, und Peptide mit geeigneten Adjuvanzien wie Toll-like-Rezeptor(TLR)-Agonisten in Wasser-Öl-Emulsion. Dazu gehören der TLR-9-Agonist CpG [3] und der TLR-2-Agonist PAM3Cys [22].
Internist 2020 - 61:690-698

https://doi.org/10.1007/s00108-020-00814-z

(c) Der/die Autor(en) 2020

H.-G. Rammensee - M. W. Löffler ·

J. S. Walz - C. Bokemeyer - S. P. Haen ·

C. Gouttefangeas

Tumorvakzinierung therapeutische Vakzinierung gegen Krebs

\section{Zusammenfassung}

Tumorzellen weisen immer Veränderungen im Vergleich zu normalen Zellen auf. Die Veränderungen können vom Immunsystem erkannt werden, was zur Zerstörung der Tumorzellen durch T-Zellen führen kann. Der Erfolg der Immuncheckpointinhibition beispielsweise beim malignen Melanom hat dies eindrucksvoll gezeigt. Viele Tumorerkrankungen sprechen jedoch nicht auf eine solche Therapie an. Hier könnte eine Vakzinierung gegen Tumorantigene hilfreich sein. Allerdings waren alle Bestrebungen in den letzten 30 Jahren praktisch erfolglos. Mit den heutigen Kenntnissen besteht jedoch neue Hoffnung.

Schlüsselwörter

DNA-Vakzinen · RNA-Vakzinen .

Peptidvakzinen - Tumorantigene .

Immunologische Adjuvanzien

Tumor vaccines-therapeutic vaccination against cancer

\section{Abstract}

Tumor cells always exhibit differences to normal cells. These differences can be recognized by the immune system, enabling the destruction of tumor cells by T cells, as was impressively demonstrated by the success of immune checkpoint inhibition, e.g., in malignant melanoma. Many cancers, however, do not respond to this kind of therapy. In these cases, vaccination against tumor antigens could be very helpful. Nevertheless, all of the efforts made in this respect during the past 30 years have been virtually futile. With current knowledge and technology there is new hope.

\section{Keywords}

Vaccines, DNA - Vaccines, RNA - Vaccines, peptide · Antigens, neoplasm · Adjuvants, immunologic 

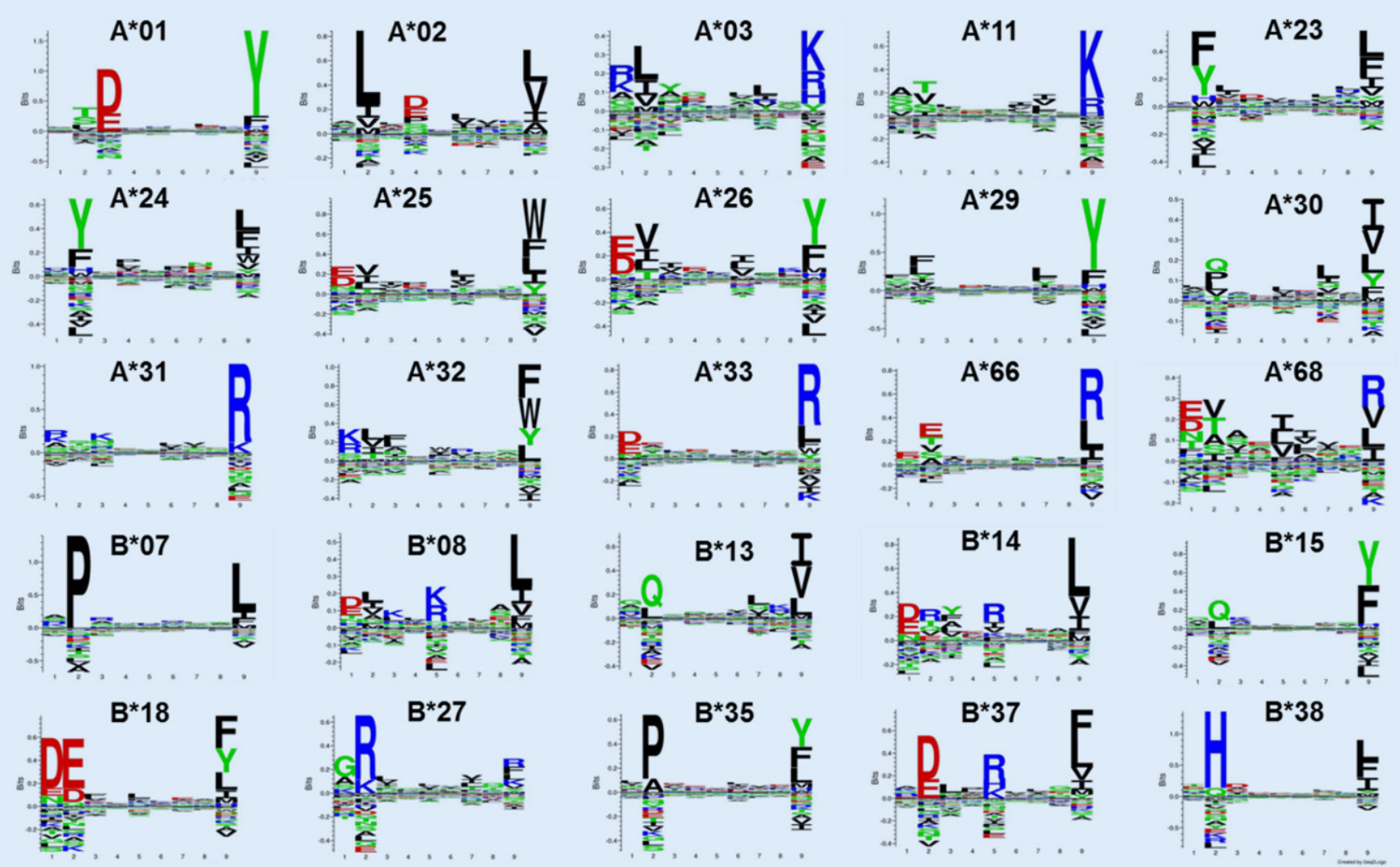

Abb. 2 \ Frequenz unterschiedlicher Aminosäuren abhängig vom entsprechenden HLA-Klasse-I-Allel. Die Größe der im EinBuchstaben-Code angegebenen Aminosäuren korreliert mit der beobachteten Frequenz verschiedener Aminosäuren an der entsprechenden Position eines 9-mer-Peptids abhängig vom jeweils angegebenen HLA-Allel. HLA Humanes Leukozytenantigen. (Aus [8])

\section{Vorläufige Erfolgskontrolle durch Immunmonitoring}

Die Bewertung der Wirksamkeit des Tumorimpfstoffs erfolgt auf zwei Arten. Das ultimative Ziel ist, eine verbesserte oder vollständige Kontrolle des Tumorwachstums bei behandelten Patienten zu erreichen. Die Tumorkontrolle wird mithilfe routinemäßiger klinischer, laborchemischer und bildgebender Verfahren beurteilt. Eine zweite wesentliche Maßnahme ist die Beurteilung der Fähigkeit des Impfstoffs, bei den Patienten vakzinspezifische T-Zell-Antworten auszulösen, eine Vorgehensweise, die als Immunmonitoring bezeichnet wird.

Für das In-vitro-Immunmonitoring werden in regelmäßigen Abständen vor, während und nach Abschluss der Impfung Blutproben entnommen (• Abb. 3). Vorzugsweise werden periphere mononukleäre Zellen, die die T-Zellen beinhalten, aus dem Blut isoliert und zunächst kryokonserviert. Für die Mes- sung von peptidspezifischen T-Zellen stehen mehrere In-vitro-Tests zur Verfügung. Am häufigsten werden „enzymelinked immunospot assays“ (ELISPOT) und die Durchflusszytometrie angewendet, die zusammengenommen eine quantitative und qualitative Bewertung der T-Zell-Aktivität ermöglichen. Das Immunmonitoring ist entscheidend für die Bestimmung der Immunogenität des Impfstoffs (beispielsweise von Peptiden), des Einflusses verschiedener Adjuvanzien und der Aufrechterhaltung der T-Zellen über das Ende der Impfphase hinaus. Daher könnten die Ergebnisse des Immunmonitorings für klinische Entscheidungen bei einzelnen Patienten Relevanz gewinnen und sich auf das Design von Impfstoffstudien der nächsten Generation auswirken. Entsprechend ist es sehr wichtig, robuste und qualitativ hochwertige Assays zu etablieren, die im Laufe der Zeit stabile Ergebnisse liefern und einen angemessenen Vergleich zwischen verschiedenen Patienten und
Studien ermöglichen. In naher Zukunft könnte das Immunmonitoring als früher Biomarker mit klinisch prädiktivem Nutzen eingesetzt werden [5].

\section{Bisherige Bemühungen}

In der klinischen Medizin spielen therapeutische Vakzinen gegen Tumoren bisher keine relevante Rolle, wohingegen prophylaktische Impfungen gegen Krankheitserreger laut Robert Koch-Institut die wichtigste präventive Maßnahme der modernen Medizin darstellen. Obwohl seit Jahrzehnten im Bereich der Tumorimpfung intensiv geforscht wird, zeichnen sich noch immer keine klaren klinischen Erfolge ab und das Feld bleibt komplex und inhomogen. Dass das Immunsystem aber prinzipiell in der Lage ist, maligne Tumoren unter Kontrolle zu halten, unterstreichen die Erfolge, die mit ICI erzielt werden können. Aber auch hier ist weiterhin Raum für Verbesserungen, da ICI bislang nur bei einigen 


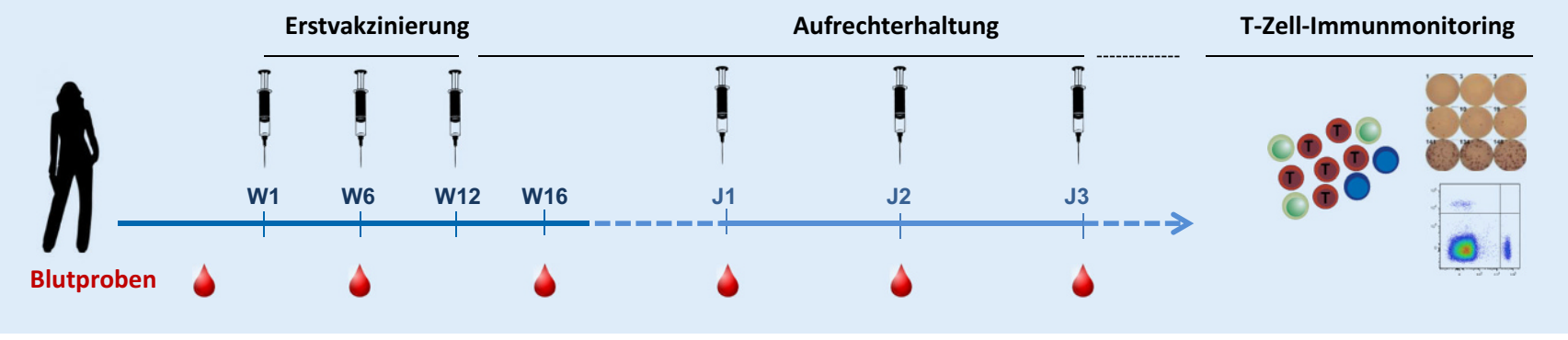

Abb. 3 ॥ Schematische Darstellung des Immunmonitorings im Verlauf einer Peptidvakzinierung. WWoche; J Jahr

Tumorentitäten und Patienten verfügbar und effektiv sind.

Als Vakzinierungsstrategien kommen wie oben beschrieben grundsätzlich sehr unterschiedliche Pattformen infrage. Die gewünschten Antigene können dabei als "genetische" Vakzinen in Form von DNA oder RNA codiert vorliegen, aber auch Peptide verschiedener Länge umfassen und zellbasiert eingesetzt werden, beispielsweise unter Verwendung viraler Vektoren oder auch beladener dendritischer Zellen. So finden sich etwa aktuelle klinische Studien, die unterschiedlich gut etablierte und standardisierte Tumorantigene wie NY-ESO-1, MART-1 und WT1 in antigenpräsentierenden Zellen verwenden [11]. Obwohl hier durchweg ein immunologisches Ansprechen berichtet wird und Patienten teilweise sogar randomisiert wurden, können die klinischen Resultate dieser zumeist frühphasigen Studien mit kleinen Fallzahlen (noch) nicht überzeugen.

\section{Auswahl der Zielantigene}

Ein Problem, das zwar grundsätzlich auf jede Tumorvakzine zutrifft, sich aber mit besonderem Nachdruck für die Peptidimpfung stellt, ist die präzise Auswahl der Zielantigene. Dabei gibt es die Möglichkeit, lediglich einzelne definierte Antigene zu selektieren oder aber eine spezielle Auswahl davon. Solche Peptide können dann als kurze Peptide, wie sie natürlicherweise auf HLA-Klasse-I-Molekülen präsentiert werden, oder aber in verlängerter Form verwendet werden, in der Annahme, dass diese dann weiter prozessiert werden und zu definierten Immunantworten führen.

Aktuell publizierte Studien zu Einzelantigenen können zwar regelhaft ein positives immunologisches Ergebnis vorweisen (Zusatzmaterial online, Tab. S1), führten jedoch häufig nicht zu einem relevanten klinischen Nutzen [28]. Viele Faktoren sprechen darum für den Einsatz von multiplen Antigenen sowie für den kombinierten Einsatz von HLA-KlasseI- und -II-Peptiden, um eine zusätzliche $\mathrm{CD}^{+}$-T-Zell-Antwort zu induzieren. Eine Einschränkung für „kurze“ HLAKlasse-I-definierte Antigene ist der individuelle HLA-Typ des Patienten. Dies kann durch Stratifizierung der Patienten nach den entsprechenden HLA-Allotypen adressiert werden, wenn vorgefertigte Peptidvakzincocktails verwendet werden. Studien mit Multipeptidvakzinen wurden in der Vergangenheit bereits durchgeführt und erste vielversprechende Ergebnisse wurden berichtet [20, 30], insbesondere beim Nierenzellkarzinom [29]. Eine randomisierte, kontrollierte Phase-III-Studie konnte aber weder starke Immunantworten noch eine klinische Wirksamkeit belegen [24]. In der Nachschau wurde unter anderem der im Gegensatz zu früheren Studienphasen - zusätzlich verwendete Tyrosinkinaseinhibitor Sunitinib als einer der potenziellen Gründe für das Versagen der Vakzine identifiziert, da präklinische Daten zeigen, dass solche Arzneimittel die Induktion peptidspezifischer T-Zellen unterdrücken können [12].

\section{》) Welche Antigene sich beim individuellen Patienten am besten eignen, ist eine hoch aktuelle Frage}

In Vakzinierungsstudien, die den Einsatz langer synthetischer Peptide unter- suchten, konnte beispielsweise für Frühformen des Vulvakarzinoms eine Wirksamkeit berichtet werden. Hier gilt es allerdings zu beachten, dass sich diese Immunität primär gegen das humane Papillomvirus HPV-16 und damit das verursachende Virus richtet [13]. Des Weiteren wurde mit langen Peptiden versucht, eine patientenindividuelle Immunantwort gegen Tumoren zu erzielen [21]. Dabei umfassten die Zielstrukturen mutierte Bereiche, gegen die überwiegend $\mathrm{CD} 4^{+}-\mathrm{T}$ Zell-Antworten ausgelöst werden konnten, lediglich bei $16 \%$ der geimpften Peptide kam es zu CD8 ${ }^{+}$-T-Zell-Antworten. Allerdings ist die klinische Aussagekraft bei 6 behandelten Patienten mit Melanom, die teilweise zusätzlich eine ICI-Behandlung erhielten, noch eingeschränkt. Ähnliches gilt für eine Studie zu einer personalisierten RNA-basierten Vakzine, in der ebenfalls multiple vorhergesagte Antigene aus patientenindividuellen Mutationen als Zielstrukturen gewählt und in der Erstanwendung am Menschen untersucht wurden [26]. Die Studie berichtet über 13 Patienten mit Melanom und belegt jeweils vakzinspezifische T-ZellAntworten. Bei 2 Patienten konnten darüber hinaus eine Infiltration des Tumors mit den spezifischen T-Zellen und deren zytotoxisches Potenzial ex vivo nachgewiesen werden. Letztlich zeigen diese beiden vielversprechenden Studien, dass die individuelle Formulierung eines Arzneimittels als Tumorvakzine grundsätzlich möglich ist und dass sich in Tumorerkrankungen mit hoher Mutationslast wie beispielsweise beim malignen Melanom - etwa mit maschinellem Lernen [21] - möglicherweise sinnvolle Zielstrukturen definieren lassen, deren klinische Relevanz allerdings bislang nicht klar belegt ist. Da die Fallzahlen bisher gering 
sind, bleibt unklar, inwieweit diese Ansätze beispielsweise nur gemeinsam mit ICI wirksam oder Letzteren sogar überlegen sind. So lassen sich etwa massenspektrometrisch in Tumoren mit hoher Mutationslast HLA-präsentierte mutierte Peptide nachweisen [2], dies gelingt aber in Tumoren mit geringerer Mutationslast nur im Ausnahmefall [17, 19]. Daher bleibt sicherlich weiterhin die Frage hochaktuell, welche Antigene bei einzelnen Tumorentitäten und beim individuellen Patienten am besten geeignet sind [9] und wie bzw. mit welchen Adjuvanzien eine effektive Immunantwort generiert werden kann, die auch klinische Wirksamkeit zeigt.

Der Schlüssel zu einer effektiven Nutzung von Vakzinen scheint deshalb ein detailliertes Wissen über die immunologischen Grundlagen und den individuellen Tumor zu sein, zudem die Kombination mit effektiven Adjuvanzien und die Wahl geeigneter Kombinationstherapien. Außerdem ist es für das Erzielen einer Wirksamkeit essenziell, die jeweilige Therapieindikation (unter anderem auch die Therapielinie) und die zu behandelnde Tumorentität mit Bedacht zu wählen, um das körpereigene Immunsystem für diese wichtige Aufgabe effektiv zu modulieren.

\section{Ideales Vorgehen aus Sicht der Autoren}

Das im Folgenden vorgeschlagene Vorgehen zur Auswahl, Herstellung und Applikation einer personalisierten Peptidvakzine basiert auf den oben beschriebenen Voraussetzungen und Erkenntnissen der letzten Jahre, insbesondere auch auf eigenen Arbeiten der Autoren.

Den Patienten wird eine autologe Tumorprobe entnommen sowie wenn möglich auch Normalgewebe vom gleichen Organ. Die auf den HLA-Molekülen präsentierten Peptide werden per Flüssigchromatographie aufgetrennt und per Massenspektrometrie identifiziert. Mit der derzeitigen Technologie finden sich sowohl auf HLA-Klasse I als auch in vielen Fällen auf HLA-Klasse II etwa 5000 oder mehr Peptide pro Gewebeprobe $[2,7]$. Durch Vergleich der Peptide von Tumor- und Normalgewebe des jeweili- gen Patienten sowie durch Vergleich mit Peptiden von Geweben anderer Individuen aus vorhandenen Datenbanken (z.B. https://hla-ligand-atlas.org) werden dann diejenigen Peptide bestimmt, die für eine personalisierte Vakzinierung geeignet erscheinen. Da es bei diesem Vorgehen der Erfahrung der Autoren zufolge jeweils Dutzende von Peptiden gibt, die nur auf dem individuellen Tumor des Patienten gefunden werden, also tumorspezifisch sind, werden daraus Peptide nach weiteren Kriterien ausgesucht, unter anderem danach, ob eine Immunogenität der Peptide bereits zuvor nachgewiesen wurde oder ob die Peptide aus Proteinen stammen, die bekannterweise eine Rolle in tumorassoziierten Stoffwechselwegen spielen [16]. Parallel erfolgt die genetische Sequenzierung der Tumorproben mit dem Ziel, tumorspezifische Mutationen zu finden. Hierauf basierend erfolgt in Zusammenschau mit dem individuellen HLA-Typ des Patienten eine Vorhersage potenzieller Neoepitope, deren natürliche Präsentation anschließend massenspektrometrisch evaluiert wird. Falls vorhanden können solche Neoepitope die oben beschriebene Auswahl nichtmutierter Tumorantigene ergänzen.

\section{) Im Kontext der Tumorvakzi- nierung muss über geeignete Kombinationstherapien nachgedacht werden}

Anschließend wird eine Auswahl von bis zu 10 Peptiden mit Adjuvans unter Good-Manufacturing-Practice-Bedingungen hergestellt, mit einem geeigneten Träger kombiniert und dann als personalisiertes Arzneimittel („mixing kit“) an den behandelnden Arzt abgegeben. Eine klinische Studie bei Patienten mit Glioblastom zeigte kürzlich, dass ein solches Vorgehen prinzipiell möglich ist [10]. In dieser Studie wurden nach mehrmaligen Injektionen starke vakzinspezifische T-Zell-Antworten nachgewiesen. Weitere Studien, die eine patientenindividuelle Multipeptidvakzinierung basierend auf Mutationsanalysen bzw. massenspektrometrischen Analysen untersuchen, rekrutieren aktuell noch. Das vermutlich für Peptidvakzinierungen besonders gut geeignete Adjuvans XS15 [22] befindet sich derzeit in der pharmazeutischen Entwicklung und soll zeitnah in einer ersten klinischen Studie getestet werden. Gemäß der Ergebnisse der Autoren kann nach einer einmaligen Vakzinierung mit Peptiden und XS15 in dem inkompletten Freund-Adjuvans Montanide $^{\text {Ts }}$ (Seppic, Paris, Frankreich) eine starke, das heißt ex vivo direkt messbare T-Zell-Antwort erzielt werden, die für mindestens 18 Monate nachweisbar ist.

Des Weiteren sollten vor Herstellung und Verabreichung einer solchen Peptidvakzine genau der Zeitpunkt und damit die möglichen Begleittherapien während einer Peptidvakzinierung bedacht werden. Eine sinnvolle Anwendung ist nur möglich, wenn ein geeignetes Verhältnis von Effektor-T-Zellen und Zielzellen vorliegt, wie es beispielsweise im adjuvanten Setting nach einer Operation oder nach einer remissionsinduzierenden Standardtherapie mit Vorhandensein einzelner Resttumorzellen der Fall ist. In jedem Fall muss aber auch über geeignete Kombinationstherapien nachgedacht werden, die im besten Fall die Immunantwort optimieren [15].

Eine basierend auf all diesen Überlegungen entwickelte personalisierte Peptidimpfung für den individuellen Tumorpatienten hat das Potenzial, zukünftig die Induktion klinisch effektiver T-Zell-Antworten zu ermöglichen.

\section{Fazit für die Praxis}

Jeder Mensch zeigt eine individuelle
Stichprobe des zellulären Peptid-
spektrums auf den HLA-Molekülen.
Das macht ein personalisiertes Vor-
gehen erforderlich, um geeignete
tumorspezifische HLA-Peptide zu
charakterisieren.
- In klinischen Studien ist es bisher
allerdings praktisch nicht gelungen,
durch Vakzinierung effektive T-Zell-
Antworten gegen solche Peptide
hervorzurufen.
Viele Faktoren sprechen dafür statt
Einzelantigenen multiple Antigene
einzusetzen und HLA-Klasse-I- und
-II-Peptide zu kombinieren, um eine




\section{zusätzliche CD4+-T-Zell-Antwort zu induzieren. \\ - Das Immunmonitoring ist entschei- dend für die Bestimmung der Immu- nogenität des Impfstoffs, des Einflus- ses verschiedener Adjuvanzien und der längerfristigen Aufrechterhal- tung der T-Zellen. \\ - Der Schlüssel zu einer effektiven Nutzung von Vakzinen scheint ein detailliertes Wissen über die immu- nologischen Grundlagen und den individuellen Tumor zu sein. Wichtig sind zudem die Kombination mit effektiven Adjuvanzien und die Wahl geeigneter Kombinationstherapien.}

\section{Korrespondenzadresse}

Prof. Dr. H.-G. Rammensee

Interfakultäres Institut für Zellbiologie (IFIZ), Abteilung Immunologie, Eberhard Karls Universität Tübingen

Auf der Morgenstelle 15, 72076 Tübingen, Deutschland

rammensee@uni-tuebingen.de

\section{Einhaltung ethischer Richtlinien}

Interessenkonflikt. H.-G. Rammensee weist auf folgende Beziehung(en) hin:S.P. Haen, M.W. Löffler, J.S. Walz und H.-G. Rammensee sind Erfinder von Patenten, die immatics Biotechnologies $\mathrm{GmbH}$ gehören. H.-G. Rammensee ist Gründer und Mitglied des wissenschaftlichen Beirats der Biotechnologieunternehmen immatics $\mathrm{GmbH}$, CureVac AG und Synimmune GmbH sowie beteiligt an einer Patentanmeldung für das Adjuvans XS15. M.W. Löffler, J.S. Walz, C. Bokemeyer, S.P. Haen und C. Gouttefangeas geben an, dass kein Interessenkonflikt besteht.

Für diesen Beitrag wurden von den Autoren keine Studien an Menschen oder Tieren durchgeführt. Für die aufgeführten Studien gelten die jeweils dort angegebenen ethischen Richtlinien.

Open Access. Dieser Artikel wird unter der Creative Commons Namensnennung 4.0 International Lizenz veröffentlicht, welche die Nutzung, Vervielfältigung, Bearbeitung, Verbreitung und Wiedergabe in jeglichem Medium und Format erlaubt, sofern Sie den/die ursprünglichen Autor(en) und die Quelle ordnungsgemäß nennen, einen Link zur Creative Commons Lizenz beifügen und angeben, ob Änderungen vorgenommen wurden.

Die in diesem Artikel enthaltenen Bilder und sonstiges Drittmaterial unterliegen ebenfalls der genannten Creative Commons Lizenz, sofern sich aus der Abbildungslegende nichts anderes ergibt. Sofern das betreffende Material nicht unter der genannten Creative Commons Lizenz steht und die betreffende Handlung nicht nach gesetzlichen Vorschriften erlaubt ist, ist für die oben aufgeführten Weiterverwendungen des Ma- terials die Einwilligung des jeweiligen Rechteinhabers einzuholen.

Weitere Details zur Lizenz entnehmen Sie bitte der Lizenzinformation auf http://creativecommons.org/ licenses/by/4.0/deed.de.

\section{Literatur}

1. Alberer M, Gnad-Vogt U, Hong HS, Mehr KT, Backert L, Finak G, Gottardo R, Bica MA, Garofano A, Koch SD, Fotin-Mleczek M, Hoerr I, Clemens R, von Sonnenburg $F$ (2017) Safety and immunogenicity of a mRNA rabies vaccine in healthy adults: an open-label, non-randomised, prospective, first-inhuman phase 1 clinical trial. Lancet 390:1511-1520

2. Bassani-Sternberg M, Bräunlein E, Klar R, Engleitner T, Sinitcyn P, Audehm S, Straub M, Weber J, Slotta-Huspenina J, Specht K, Martignoni ME, Werner A, Hein R, Busch DH, Peschel C, Rad R, Cox J, Mann M, Krackhardt AM (2016) Direct identification of clinically relevant neoepitopes presented on native human melanoma tissue by mass spectrometry. Nat Commun 7:13404

3. Baumgaertner P, Jandus C, Rivals JP, Derre L, Lovgren T, Baitsch L, Guillaume P, Luescher IF, Berthod G, Matter M, Rufer N, Michielin O, Speiser DE (2012) Vaccination-induced functional competence of circulating human tumor-specific CD8 T-cells. Int J Cancer 130:2607-2617

4. Boegel S, Castle JC, Kodysh J, O'Donnell T, Rubinsteyn A (2019) Bioinformatic methods for cancer neoantigen prediction. Prog Mol Biol Transl Sci 164:25-60

5. Britten CM, van der Burg SH, Gouttefangeas C (2015) A framework for T cell assays. Oncotarget 6:35143-35144

6. Chabanon RM, Pedrero M, Lefebvre C, Marabelle A, Soria JC, Postel-Vinay S (2016) Mutational landscape and sensitivity to immune checkpoint blockers. Clin Cancer Res 22:4309-4321

7. Freudenmann LK, Marcu A, Stevanović S (2018) Mapping the tumour human leukocyte antigen (HLA) ligandome by mass spectrometry. Immunology 154:331-345

8. Ghosh M, Di Marco M, Stevanović S (2019) Identification of MHC ligands and establishing MHC class I peptide motifs. Methods Mol Biol 1988:137-147

9. Gubin MM, Schreiber RD (2015) The odds of immunotherapy success. Science 350:158-159

10. Hilf N, Kuttruff-Coqui S, Frenzel K, Bukur V, Stevanović S, Gouttefangeas C, Platten M, Tabatabai G, Dutoit V, van der Burg SH, Thor Straten P, Martínez-Ricarte F, Ponsati B, Okada $\mathrm{H}_{\text {, }}$ Lassen $U$, Admon A, Ottensmeier $\mathrm{CH}$, Ulges A, Kreiter S, von Deimling A, Skardelly M, Migliorini D, Kroep JR, Idorn M, Rodon J, Piró J, Poulsen HS, Shraibman B, McCann K, Mendrzyk R, Löwer M, Stieglbauer M, Britten CM, Capper D, Welters MJP Sahuquillo J, Kiesel K, Derhovanessian E, Rusch E, Bunse L, Song C, Heesch S, Wagner C, KemmerBrück A, Ludwig J, Castle JC, Schoor O, Tadmor AD Green E, Fritsche J, Meyer M, Pawlowski N, Dorner S Hoffgaard F, Rössler B, Maurer D, Weinschenk T, Reinhardt C, Huber C, Rammensee HG, SinghJasuja H, Sahin U, Dietrich PY, Wick W (2019) Actively personalized vaccination trial for newly diagnosed glioblastoma. Nature 565:240-245

11. Ito Z, Kan S, Bito T, Horiuchi S, Akasu T, Yoshida S, Kajihara M, Hokari A, Saruta M, Yoshida N, Kobayashi M, Ohkusa T, Shimodaira S, Okamoto M, Sugiyama H, Koido S (2019) Predicted markers of overall survival in pancreatic cancer patients receiving dendritic cell vaccinations targeting WT1. Oncology 97:135-148

12. Jaini R, Rayman P, Cohen PA, Finke JH, Tuohy VK (2014) Combination of sunitinib with antitumor vaccination inhibits $T$ cell priming and requires careful scheduling to achieve productive immunotherapy. Int J Cancer 134:1695-1705

13. Kenter GG, Welters MJ, Valentijn AR, Lowik MJ, Berends-van der Meer DM, Vloon AP, Essahsah F, Fathers LM, Offringa R, Drijfhout JW, Wafelman AR, OostendorpJ,Fleuren GJ, van derBurg SH, MeliefC (2009) Vaccination against HPV-16 oncoproteins for vulvar intraepithelial neoplasia. N Engl J Med 361:1838-1847

14. Kowalewski DJ, Schuster H, Backert L, Berlin C, Kahn S, Kanz L, Salih HR, Rammensee HG, Stevanovic S, Stickel JS (2015) HLA ligandome analysis identifies the underlying specificities of spontaneous antileukemia immune responses in chronic lymphocytic leukemia (CLL). Proc Natl Acad SciU SA 112:E166-E175

15. Kowalewski DJ, Walz $S$, Backert $L$, Schuster $H$, Kohlbacher O, Weisel K, Rittig SM, Kanz L, Salih HR, Rammensee HG, Stevanović S, Stickel JS (2016) Carfilzomib alters the HLA-presented peptidome of myeloma cells and impairs presentation of peptides with aromatic C-termini. Blood Cancer J 6:e411

16. Löffler MW, Kowalewski DJ, Backert L, Bernhardt J, Adam P, Schuster H, Dengler F, Backes D, Kopp HG, Beckert S, Wagner S, Königsrainer I, Kohlbacher O, Kanz L, Königsrainer A, Rammensee HG, Stevanović S, Haen SP (2018) Mapping the HLA ligandome of colorectal cancer reveals an imprint of malignant cell transformation. Cancer Res 78:4627-4641

17. Löffler MW, Mohr C, Bichmann L, Freudenmann LK, Walzer M, Schroeder CM, Trautwein N, Hilke FJ, Zinser RS, Mühlenbruch L, Kowalewski DJ, Schuster H, Sturm M, Matthes J, Riess O, Czemmel S, Nahnsen S, Königsrainer I, Thiel K, Nadalin S, Beckert S, Bösmüller $H$, Fend $F$, Velic A, Maček B, Haen SP, Buonaguro L, Kohlbacher O, StevanovićS, Königsrainer A, Rammensee HG (2019) Multiomics discovery of exome-derived neoantigens in hepatocellular carcinoma. Genome Med 11:28

18. Marijt KA, Blijleven L, Verdegaal EME, Kester MG, Kowalewski DJ, Rammensee HG, Stevanović S, Heemskerk MHM, van der Burg SH, van Hall T (2018) Identification of non-mutated neoantigens presented by TAP-deficient tumors. J Exp Med 215:2325-2337

19. Newey A, Griffiths B, Michaux J, Pak HS, Stevenson BJ, Woolston A, Semiannikova M, Spain G, Barber LJ, Matthews N, Rao S, Watkins D, Chau I, Coukos G, Racle J, Gfeller D, Starling N, Cunningham D, Bassani-Sternberg M, Gerlinger M (2019) Immunopeptidomics of colorectal cancer organoids reveals a sparse HLA class I neoantigen landscape and no increase in neoantigens with interferon or MEK-inhibitor treatment. JImmunother Cancer 7:309

20. Noguchi M, Matsumoto K, Uemura H, Arai G, Eto $M$, Naito S, Ohyama C, Nasu Y, Tanaka M, Moriya F, Suekane S, Matsueda S, Komatsu N, Sasada T, Yamada A, Kakuma T, Itoh K (2016) An open-label, randomized phase II trial of personalized peptide vaccination in patients with bladder cancer that progressed after platinum-based chemotherapy. Clin Cancer Res 22:54-60

21. Ott PA, Hu Z, Keskin DB, Shukla SA, Sun J, Bozym DJ, Zhang W, Luoma A, Giobbie-Hurder A, Peter L, Chen C, Olive O, Carter TA, Li S, Lieb DJ, Eisenhaure T, Gjini E, Stevens J, Lane WJ, Javeri I, 
Nellaiappan K, Salazar AM, Daley H, Seaman M Buchbinder El, Yoon $\mathrm{CH}$, Harden $\mathrm{M}$, Lennon $\mathrm{N}$, Gabriel S, Rodig SJ, Barouch DH, Aster JC, Getz G, Wucherpfennig K, Neuberg D, Ritz J, Lander ES, Fritsch EF, Hacohen N, Wu CJ (2017) An immunogenic personal neoantigen vaccine for patients with melanoma. Nature 547:217-221

22. Rammensee HG, Wiesmüller KH, Chandran PA Zelba H, Rusch E, Gouttefangeas C, Kowalewski DJ, Di Marco M, Haen SP, Walz JS, Gloria YC, Bödder J, Schertel JM, Tunger A, Müller L, Kiessler $M$, Wehner R, Schmitz M, Jakobi M, SchneiderhanMarra N, Klein R, Laske K, Artzner K, Backert L, Schuster H, Schwenck J, Weber ANR, Pichler BJ, Kneilling $M$, la Fougère $C$, Forchhammer $S$, Metzler G, Bauer J, Weide B, Schippert W, Stevanović S, Löffler MW (2019) A new synthetic toll-like receptor $1 / 2$ ligand is an efficient adjuvant for peptide vaccination in a human volunteer. JImmunother Cancer 7:307

23. ReinhardK, RengstlB, OehmP,MichelK, BillmeierA HaydukN, Klein O, Kuna K, Ouchan Y, WöllS, ChristE, Weber D, Suchan M, Bukur T, Birtel M, Jahndel V, Mroz K, Hobohm K, Kranz L, Diken M, Kühlcke K, Türeci O, Sahin U (2020) An RNA vaccine drives expansion and efficacy of claudin-CAR-T cells against solid tumors. Science 367(6476):446-453

24. Rini BI, Stenzl A, Zdrojowy R, Kogan M, Shkolnik M, Oudard S, Weikert S, Bracarda S, Crabb SJ, Bedke J, Ludwig J, Maurer D, Mendrzyk R, Wagner C, Mahr A, Fritsche J, Weinschenk T, Walter $S$, Kirner A, Singh-Jasuja $\mathrm{H}$, Reinhardt $\mathrm{C}$, Eisen $\mathrm{T}$ (2016) IMA901, a multipeptide cancer vaccine, plus sunitinib versus sunitinib alone, as first-line therapy for advanced or metastatic renal cell carcinoma (IMPRINT): a multicentre, open-label, randomised, controlled, phase 3 trial. Lancet Oncol 17:1599-1611

25. Robinson J, Halliwell JA, Hayhurst JD, Flicek $P$, Parham P, Marsh SG (2015) The IPD and IMGT/HLA database: allele variant databases. Nucleic Acids Res 43:D423-D431

26. Sahin U, Derhovanessian E, Miller M, Kloke BP, Simon $P$, Löwer $M$, Bukur V, Tadmor AD, Luxemburger $\mathrm{U}$, Schrörs B, Omokoko T, Vormehr $\mathrm{M}$, Albrecht C, Paruzynski A, Kuhn AN, Buck J, Heesch S, Schreeb KH, Müller F, Ortseifer I, Vogler I, Godehardt E, Attig S, Rae R, Breitkreuz A, Tolliver C, Suchan M, Martic G, Hohberger A, Sorn P, Diekmann J, Ciesla J, Waksmann O, Brück AK, Witt M, Zillgen M, Rothermel A, Kasemann B, Langer D, Bolte S, Diken M, Kreiter S, Nemecek R, Gebhardt C, Grabbe S, Höller C, Utikal J, Huber C, Loquai C, Türeci O (2017) Personalized RNA mutanome vaccines mobilize poly-specific therapeutic immunity against cancer. Nature 547:222-226

27. Schuster H, Peper JK, Bösmüller HC, Röhle K, Backert L, Bilich T, Ney B, Löffler MW, Kowalewski DJ, Trautwein N, Rabsteyn A, Engler T, Braun S, Haen SP, Walz JS, Schmid-Horch B, Brucker SY, Wallwiener D, Kohlbacher O, Fend F, Rammensee HG, Stevanović S, Staebler A, Wagner $P$ (2017) The immunopeptidomic landscape of ovarian carcinomas. Proc Natl Acad Sci U S A 114:E9942-E9951

28. Shima H, Tsurita G, Wada S, Hirohashi Y, Yasui $H$, Hayashi $H$, Miyakoshi T, Watanabe K, Murai A, Asanuma $\mathrm{H}$, Tokita $\mathrm{S}$, Kubo T, Nakatsugawa $\mathrm{M}$ Kanaseki T, Tsukahara T, Nakae $Y$, Sugita $O$, Ito YM, Ota Y, Kimura Y, Kutomi G, Hirata K, Mizuguchi T, Imai K, Takemasa I, Sato N, Torigoe T (2019) Randomized phase II trial of survivin 2B peptide vaccination for patients with HLA-A24- positive pancreatic adenocarcinoma. Cancer Sci 110:2378-2385

29. Walter S, Weinschenk T, Stenzl A, Zdrojowy R, Pluzanska A, Szczylik C, Staehler M, Brugger W, Dietrich PY, Mendrzyk R, Hilf N, Schoor O, Fritsche J, Mahr A, Maurer D, Vass V, Trautwein C, Lewandrowski P, Flohr C, Pohla H, Stanczak JJ, Bronte V, Mandruzzato S, Biedermann T, PawelecG, Derhovanessian E, Yamagishi H, Miki T, Hongo F, Takaha N, Hirakawa K, Tanaka H, Stevanovic S, Frisch J, Mayer-Mokler A, Kirner A, Rammensee HG, Reinhardt C, Singh-Jasuja H (2012) Multipeptide immune response to cancer vaccine IMA901 after single-dose cyclophosphamide associates with longer patient survival. Nat Med 18:1254-1261

30. Yoshimura K, Minami T, Nozawa M, Kimura T, Egawa S, Fujimoto H, Yamada A, Itoh K, Uemura H (2016)A phase 2 randomized controlled trial of personalized peptide vaccine immunotherapy with low-dose dexamethasone versus dexamethasone alone in chemotherapy-naive castration-resistant prostate cancer. Eur Urol 70:35-41
Sicherheit bei der Einnahme von immunsupprimierenden Medikamenten

Studie zu COVID-19 bei Rheuma

Wissenschaftler sind der Frage nachgegangen, inwieweit die unterschiedlichen Medikamentengruppen die Wahrscheinlichkeit für einen Krankenhausaufenthalt bei RheumaKranken mit einer COVID-19-Infektion erhöhen.

Hierfür wurde eine Fallserie von Personen mit rheumatischen Erkrankungen und COVID-19 aus dem „EULAR and Global Rheumatology Alliance COVID-19"Register analysiert. Insgesamt gingen 600 Fälle aus 40 Ländern in die Studie ein. Die Forscher analysierten Alter, Geschlecht, Raucherstatus, Diagnose rheumatischer Erkrankungen, Komorbiditäten und Medikamente gegen rheumatische Erkrankungen, die unmittelbar vor der Infektion eingenommen wurden.

Die Einnahme konventioneller krankheitsmodifizierender Antirheumatika (csDMARDs) - wie Anti-Malaria-Mittel oder Medikamente aus der Krebstherapie allein oder in Kombination mit Biologika oder die Einnahme von nichtsteroidale Antirheumatika (NSAR) war nicht mit einem Krankenhausaufenthalt assoziiert. Die Einnahme von TNF alpha-Hemmern war mit einer verringerten Wahrscheinlichkeit eines Krankenhausaufenthalts verbunden, während kein Zusammenhang mit der Einnahme von Malariamitteln beobachtet wurde. Eine Behandlung mit mehr als $10 \mathrm{mg}$ Prednison pro Tag das entspricht einer mäßigen bis hohen Kortisondosis - war mit einer höheren Wahrscheinlichkeit eines Klinikaufenthalts verbunden. Prednison ist ein Glukokortikoid, das in der Rheumatologie häufig als schnell wirksamer Entzündungshemmer eingesetzt wird.

Gianfrancesco M., Hyrich Kl., Al-Adely S. et al. Ann Rheum Dis 2020. [epub ahead of print.] doi:10.1136/ annrheumdis-2020217871. 
Hier steht eine Anzeige.

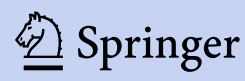

